\title{
Taking airway disease seriously
}

\section{Pierre Ernst}

Sadatsafavi and colleagues address the question of the safety of long-acting $\beta$-agonists (LABA) in asthma with and without the concomitant use of inhaled corticosteroids (ICS). ${ }^{1}$ They carried out a study within the administrative healthcare databases of the province of British Columbia, Canada. The universal healthcare system provides healthcare for all residents of the province and includes coverage of prescription drugs. The results of the study are therefore based on a complete population avoiding the exaggeration of benefit and the minimisation of harm that may result in randomised controlled trials (RCTs) which often exclude those with comorbid disease. Within a cohort of more than 120000 subjects, aged between 12 years and 45 years at study initiation, who were users of asthma medications, they identified more than 3000 hospitalisations for asthma. They then compared the medication used in the 12 months prior to the asthma hospitalisation with the use of asthma medications among control subjects with similar asthma severity that had not been hospitalised at that time.

Several of the results reported are of interest. First, irregular use of maintenance asthma therapy with ICS or the combination ICS/LABA is substantially more common than regular use. This is not new, ${ }^{2}$ but is particularly troublesome for the ICS/LABA combination since it is only clearly indicated in subjects who do not achieve control of their asthma with regular ICS use. Second, the risk of an asthma hospitalisation, which may be seen as an indicator of a severe asthma exacerbation, is increased with the regular use of LABA without concomitant regular use of an ICS. This result is consistent with meta-analyses of RCTs which also suggest an increase in asthma mortality with this treatment strategy. ${ }^{3} 4$ One may therefore hope that the use of LABA alone in the treatment of asthma has become a strategy to be avoided.

Correspondence to Dr Pierre Ernst, Centre for Clinical Epidemiology, Lady Davis Research Institute for Medical Research, Jewish General Hospital, McGill University, 3755 Cote Ste-Catherine, Montreal, Quebec, Canada H3T 1E2; pierre.ernst@mcgill.ca
The most important result reported by Sadatsafavi and colleagues is that the risk of an asthma hospitalisation is similar among regular users of ICS/LABA or of ICS alone suggesting no excess in the risk of severe asthma exacerbations when LABAs are used in conjunction with an ICS. The simplest way to achieve this is to use an ICS/LABA combination in a single inhaler. This equality in risk of severe exacerbations was found despite the fact that patients receiving an ICS/LABA combination likely had more severe asthma and therefore a higher risk of an asthma hospitalisation than subjects on ICS alone even after adjusting for severity using the incomplete indicators of severity available in administrative databases. The answer provided by this study is consistent with that obtained by properly done meta-analyses of RCTs where the use of ICS in combination with a LABA was mandated. ${ }^{3} 4$

Therefore, why is the safety of ICS/ LABA in combination sufficiently controversial that the latest US Federal Drug Administration safety warnings suggest using higher doses of ICS before using an ICS/LABA combination and to revert to ICS alone as soon as asthma control is achieved? Furthermore, why are large RCTs of the safety of ICS/LABA currently ongoing? One logical reason might be that the excess mortality from asthma clearly seen with use of LABAs in asthma may still occur with ICS/LABA combinations and that meta-analyses of RCTs as well as observational studies such as that by Sadatsafavi and colleagues are underpowered to detect this excess mortality. For an excess of asthma deaths without an excess of asthma hospitalisations to be plausible, however, asthma deaths would have to have a different pathophysiology than severe asthma exacerbations leading to hospitalisations. While it is possible that use of bronchodilators may delay hospitalisation and increase the possibility of an out-of-hospital death, the more likely scenario is a continuum from poor control to severe exacerbation leading occasionally to death when particular situations, such as access to emergency services, social circumstance or quality of care intervene. ${ }^{5}$ The continuum from severe exacerbations to asthma mortality is further re-enforced by the prevention of asthma hospitalisations and death with ICS. $^{6}$

The difficulty at the moment and for the future is that the RCTs comparing the safety of ICS/LABA combinations with ICS alone are unlikely to provide a clear answer on the risk of mortality as recently pointed out by Suissa, ${ }^{7}$ while the evidence for severe exacerbations and asthma control favour the ICS/LABA combinations over ICS alone. ${ }^{4}$ Thus, I would have to agree with Sears, ${ }^{3}$ that these studies may be futile and a waste of limited resources. It might be worth considering carrying out multiple concurrent studies such as the one by Sadatsafavi and colleagues and combining their results so as to provide sufficient power to examine mortality. $^{8}$

What might one do now to improve the safety and effectiveness of asthma therapy? My first choice would be to target interventions that might improve compliance with asthma maintenance therapy, especially ICS. This would require convincing primary care physicians that regular therapy is advantageous in persistent asthma which in turn requires tools to distinguish intermittent and persistent disease. ${ }^{9}$

For persistent asthma, it at first appears tempting to only allow LABAs to be prescribed as ICS/LABA combinations. These medications are also used for the treatment of COPD, however, and such a policy would further encourage the indiscriminate use of ICS/LABA combinations in COPD. This is undesirable given the weakness of the evidence of effectiveness of ICS in COPD, even as ICS/LABA combinations, ${ }^{10}$ and the strong evidence of adverse effects of ICS in patients with COPD, especially severe pneumonia associated with a significant excess mortality. ${ }^{11} 12$

The reality of asthma and COPD therapy today, at least in Canada, is that patients are being treated without a firm diagnosis and without assessment of individual needs, ${ }^{13-15}$ while ICS/LABA combinations are being used indiscriminately in patients with respiratory symptoms. ${ }^{16} 17$ This one size fits all approach has been facilitated by the pharmaceutical industry dictating the research agenda and by physicians choosing the path of least resistance.

How might we improve things? Research is needed into what might be done to support primary care physicians in the treatment of airway disease. ${ }^{9}$ I 
mentioned earlier, providing first-line physicians with tools to recognise significant airway disease (spirometry with bronchodilator response ${ }^{18}$ ) and the ability to distinguish asthma from COPD (diagnostic algorithms, FeNO ${ }^{19}$ ). For the specialist physician, the analysis of sputum inflammatory profiles provides the best currently available way to predict response to therapy. ${ }^{20}$ This is the only currently available method which reliably allows the correct treatment to be administered to patients in whom the diagnosis is unclear; for example, the apparently allergic asthma not responding to ICS or the patient with COPD with frequent exacerbations despite long-acting bronchodilators. Sputum examination remains underused except in a small number of centres. To me, this is equivalent to treating lung cancer without a firm histological diagnosis. When are we going to start taking airway disease seriously?

\section{Competing interests None.}

Provenance and peer review Not commissioned; internally peer reviewed.

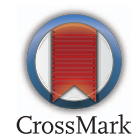

To cite Ernst P. Thorax 2014;69:307-308.

Published Online First 7 January 2014

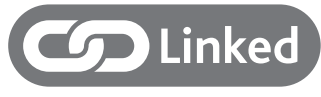

- http://dx.doi.org/10.1136/thoraxjnl-2013-203998

Thorax 2014;69:307-308.

doi:10.1136/thoraxjnl-2013-204709

\section{REFERENCES}

1 Sadatsafavi M, Lynd LD, Marra CA. Dispensation of long-acting $\beta$ agonists with or without inhaled corticosteroids, and risk of asthma-related hospitalisation: a population-based study. Thorax 2014;69:328-34.

2 Lacasse $\mathrm{Y}$, Archibald $\mathrm{H}$, Ernst $\mathrm{P}$, et al. Patterns and determinants of compliance with inhaled steroids in adults with asthma. Can Respir J 2005;12:211-17.

3 Sears MR. The FDA-mandated trial of safety of long-acting beta-agonists in asthma: finality or futility? Thorax 2013;68:195-8.

4 Rodrigo GJ, Castro-Rodriguez JA. Safety of long-acting beta agonists for the treatment of asthma: clearing the air. Thorax 2012;67:342-9.

5 Rea HH, Sears MR, Beaglehole R, et al. Lessons from the national asthma mortality study: circumstances surrounding death. NZ Med J 1987;100:10-13.

6 Suissa S, Ernst P. Inhaled corticosteroids: impact on asthma morbidity and mortality. J Allergy Clin Immunol 2001;107:937-44.

7 Suissa S, Ariel A. US Food and Drug Administration-mandated trials of long-acting beta-agonists safety in asthma: will we know the answer? Chest 2013;143:1208-13.

8 Suissa S, Henry D, Caetano P, et al. CNODES: the Canadian Network for Observational Drug Effect Studies. Open Med 2012;6:e134-40.

9 Lamontagne J, Pelaez S, Grad R, et al. Facilitators and solutions for practicing optimal guided asthma self-management: The physician perspective. Can Respir J 2013;20:285-93.
10 Suissa $\mathrm{S}$, Ernst $\mathrm{P}$, Vandemheen $\mathrm{KL}$, et al. Methodological issues in therapeutic trials of COPD. Eur Respir J 2008;31:927-33.

11 Suissa S. Number needed to treat in COPD: exacerbations versus pneumonias. Thorax 2013;68:540-3.

12 Ernst $\mathrm{P}$, Gonzalez AV, Brassard P, et al. Inhaled corticosteroid use in chronic obstructive pulmonary disease and the risk of hospitalization for pneumonia. Am J Respir Crit Care Med 2007;176:162-6.

13 Boulet LP, Bourbeau J, Skomro R, et al. Major care gaps in asthma, sleep and chronic obstructive pulmonary disease: a road map for knowledge translation. Can Respir J 2013;20: 265-9.

14 Gershon AS, Victor JC, Guan J, et al. Pulmonary function testing in the diagnosis of asthma: a population study. Chest 2012;141:1190-6.

15 Gershon AS, Hwee J, Croxford R, et al. Patient and physician factors associated with pulmonary function testing for chronic obstructive pulmonary disease (COPD): a population study. Chest Published Online First: 5 Sept 2013. doi:10.1378/chest.13-0790

16 Breton MC, LeLorier J, Forget $A$, et al. Use of combination therapy in asthma: are they prescribed according to guidelines. Respir Med 2007;101:1916-23.

17 Hernandez P, Balter MS, Bourbeau J, et al. Canadian practice assessment in chronic obstructive pulmonary disease: respiratory specialist physician perception versus patient reality. Can Respir J 2013;20:97-105.

18 Coates AL, Graham BL, McFadden RG, et al. Spirometry in primary care. Can Respir J 2013;20:13-21.

19 Price D, Ryan D, Burden A, et al. Using fractional exhaled nitric oxide (FeNO) to diagnose steroid-responsive disease and guide asthma management in routine care. Clin Trans/ Allergy 2013;3:37.

20 Nair P. Update on clinical inflammometry for the management of airway diseases. Can Respir J 2013;20:117-20. 